\title{
A young patient with severe virilization, hypertension and bilateral adrenal hyperplasia
}

\author{
S A Abhayaratna ${ }^{1}$, N P Somasundaram ${ }^{2}$
}

Sri Lanka Journal of Diabetes, Endocrinology and Metabolism 2013; 3: 91-94

\begin{abstract}
We describe an unusual late presentation of $11 \beta$-hydroxylase deficiency in a severely virilized, 23 year old patient, who presented with intracerebral haemorrhage, hypertension and short stature. The patient was raised as a male but had hypospadias, absent testicles from birth and had precocious puberty during his childhood. Plasma testosterone level was elevated $(16 \mathrm{ng} / \mathrm{mL})$ with suppressed $\mathrm{FSH}$ and $\mathrm{LH}$ levels (<1 U/L). Basal 17-hydoxyprogesterone was markedly elevated (>19.2 ng/mL-ref range: 0.5-2.1 $\mathrm{ng} / \mathrm{mL}$ ). CT scan abdomen showed bilateral marked adrenal hyperplasia with mullerian structures and karyotyping showed $46 \mathrm{XX}$ with negative sex-determining region $\mathrm{Y}(\mathrm{SRY})$ test. A clinical diagnosis of $11 \beta$ hydroxylase deficiency was made in view of hypertension with severe virilization in a $46 X X$ individual. The patient was managed with antihypertensive drugs, monitoring of serum testosterone and 17hydroxyprogesterone levels since she the patient decided to remain as a male.
\end{abstract}

Key words: congenital adrenal hyperplasia, $11-\beta$-hydroxylase deficiency.

\section{Introduction}

Congenital adrenal hyperplasia (CAH) is a family of disorders, with an autosomal recessive inheritance, characterized by enzymatic defects in one of the steps in cortisol production. Steroid $11 \beta$-hydroxylase deficiency is the second most common cause of $\mathrm{CAH}$, accounting for $5-8 \%$ of all cases $(1,2)$. It is caused by the mutation of the CYP11B1 gene that encodes the enzyme. In classical $11 \beta$-hydroxylase deficiency, mutations of the CYP11B1 gene result in decreased activity or inactivation of the enzyme (3). Deficiency of $11 \beta$-hydroxylation causes a decrease in conversion of 11-deoxycorticosterone to corticosterone and 11-deoxycortisol to cortisol. (Figure 1). Reduced cortisol feedback gives rise to an increase in adrenocorticotropin

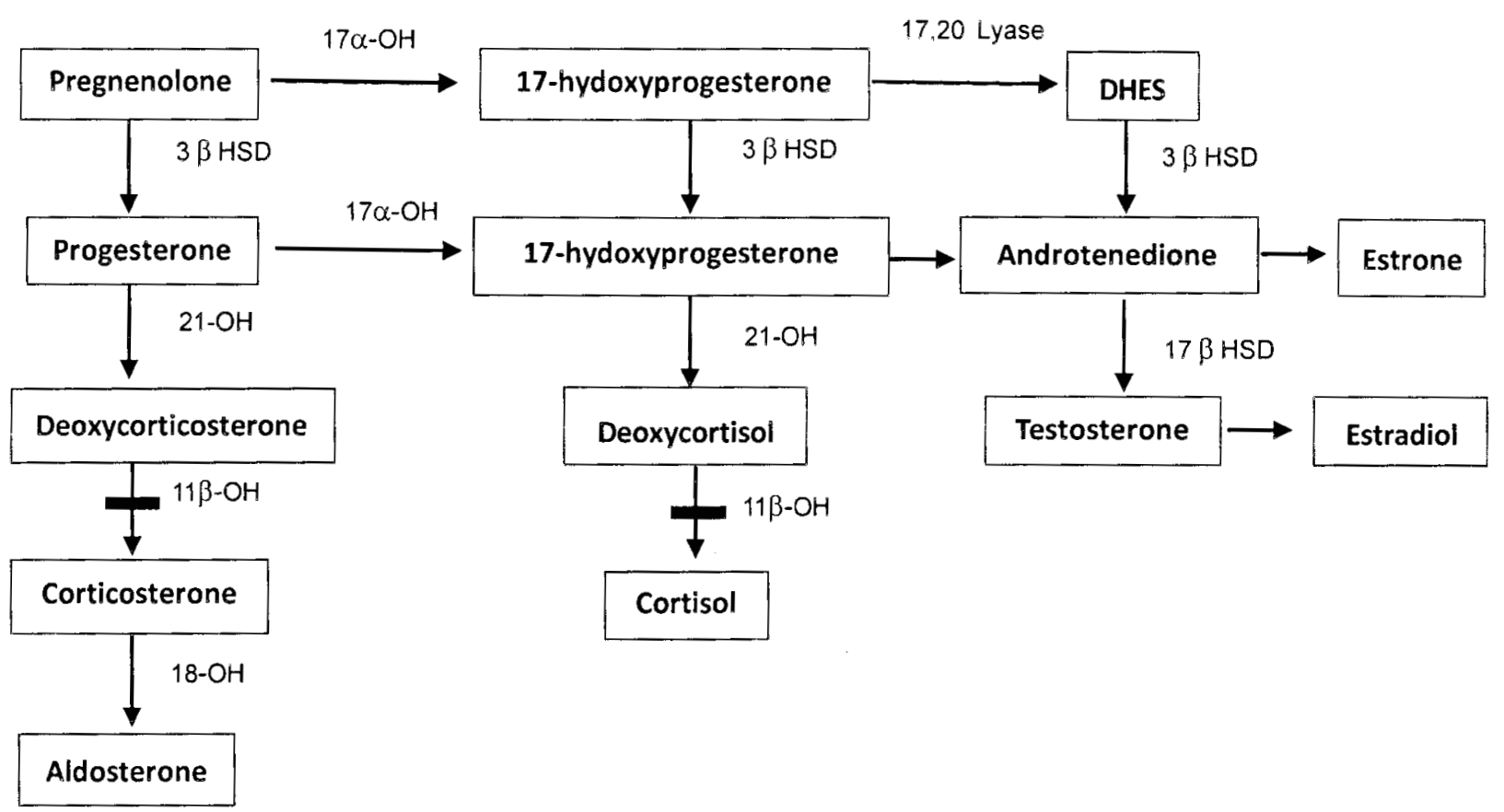

Figure 1. Adrenal steroid synthesis. Deficiency in 11 $\beta$-hydroxylase (CYP11B1) results in accumulation of DOC and consequent hypertension; increased androgen synthesis.

${ }^{1}$ Senior Registrar in Endocrinology, ${ }^{2}$ Consultant Endocrinologist, National Hospital of Sri Lanka, Colombo. 
(ACTH) secretion, which in turn leads to overproduction of precursors proximal to the enzyme defect. These precursors serve as substrates for the unimpeded androgen pathways, so that adrenal androgen secretion is increased (4). Commonest form of CAH is due to 21-hydroxylase deficiency. This enzyme deficiency results in decreased cortisol synthesis with elevated ACTH secretion, leading to increase adrenal androgen production. The disease is caused by mutations in the CYP21 gene encoding the steroid 21-hydroxylase enzyme. In contrast to 21-hydroxylase deficiency, untreated $11 \beta$-hydroxylase deficient patients have no salt loss. Overproduction of deoxycorticosterone and possibly other precursors with mineralocorticoid activity cause hypertension in the former condition (4).

\section{Case report}

A 23 year old male patient was admitted to the neurosurgical ward with sudden onset altered consciousness, difficulty in speech and right sided face-armleg weakness. The patient was found to have hypertension on admission (blood pressure 240/130 mmHg). A computerized tomography (CT) scan of the brain showed left sided intracerebral haemorrhage with cerebral oedema. He was transferred to the neurosurgical intensive care unit (ICU) and blood pressure was controlled with antihypertensive drugs and intravenous dexamethazone was administered for cerebral oedema. He underwent a four vessel digital subtraction angiography which was found to be normal. His condition gradually improved in the ICU and blood pressure was controlled with losartan $50 \mathrm{mg}$ twice daily and was transferred to the neurosurgical ward. During further examination in the ward he was found to have bilateral absent testicles and hypospadias apart from his short stature. His routine investigations showed mild persistent hypokalaemia (despite treatment with losartan), hypertensive heart disease and bilateral adrenal masses on ultrasound scan of the abdomen (right side: 4 $\mathrm{cm} \times 3.5 \mathrm{~cm}$, left side: $2.5 \mathrm{~cm} \times 2 \mathrm{~cm}$ ). The patient was referred for endocrine opinion and a detailed history revealed that he was assigned male sex at birth but had absent testes at the neonatal examination. However, the parent's defaulted clinic follow up. By the age of five years he was found to be taller then most of his peers and around the same time he developed deepening of voice, axillary and facial hair. He stopped gaining height since then and by the age of 10 years he was shorter than most of his peers. At this point, parents sought medical advice and after investigations (including karyotype analysis), he was informed to be of "female gender". Parents could not accept raising this child as a female and the child was lost to follow-up until he presented to us at the age of 23 years with hypertension induced cerebral hemorrhage. There was no family history of similar disease. He was $145 \mathrm{~cm}$ tall (well below 3 rd percentile) and pigmented. His pubic hair was of Tanner stage IV, with a phallus of $6.5 \mathrm{~cm}$ in length and penile hypospadias. The scrotal sac was empty and testes were not palpable in the inguinal area. Blood pressure was $160 / 100 \mathrm{mmHg}$ with grade three hypertensive retinopathy. There were no palpable masses or renal bruits in the abdomen and there was no gynaecomastia.

His plasma testosterone level was elevated $(>16 \mathrm{ng} /$ $\mathrm{mL}$ ) with suppressed FSH and LH levels ( $<1 \mathrm{U} / \mathrm{L})$. Basal 17-hydoxyprogesterone was markedly elevated $(>19.2$ $\mathrm{ng} / \mathrm{mL}$ - ref range: $0.5-2.1 \mathrm{ng} / \mathrm{mL}$ ). 11-deoxycortisol levels could not be performed due to unavailability of the test. CT scan abdomen showed bilateral marked adrenal hyperplasia with presence of mullerian structures (Figure 2) and
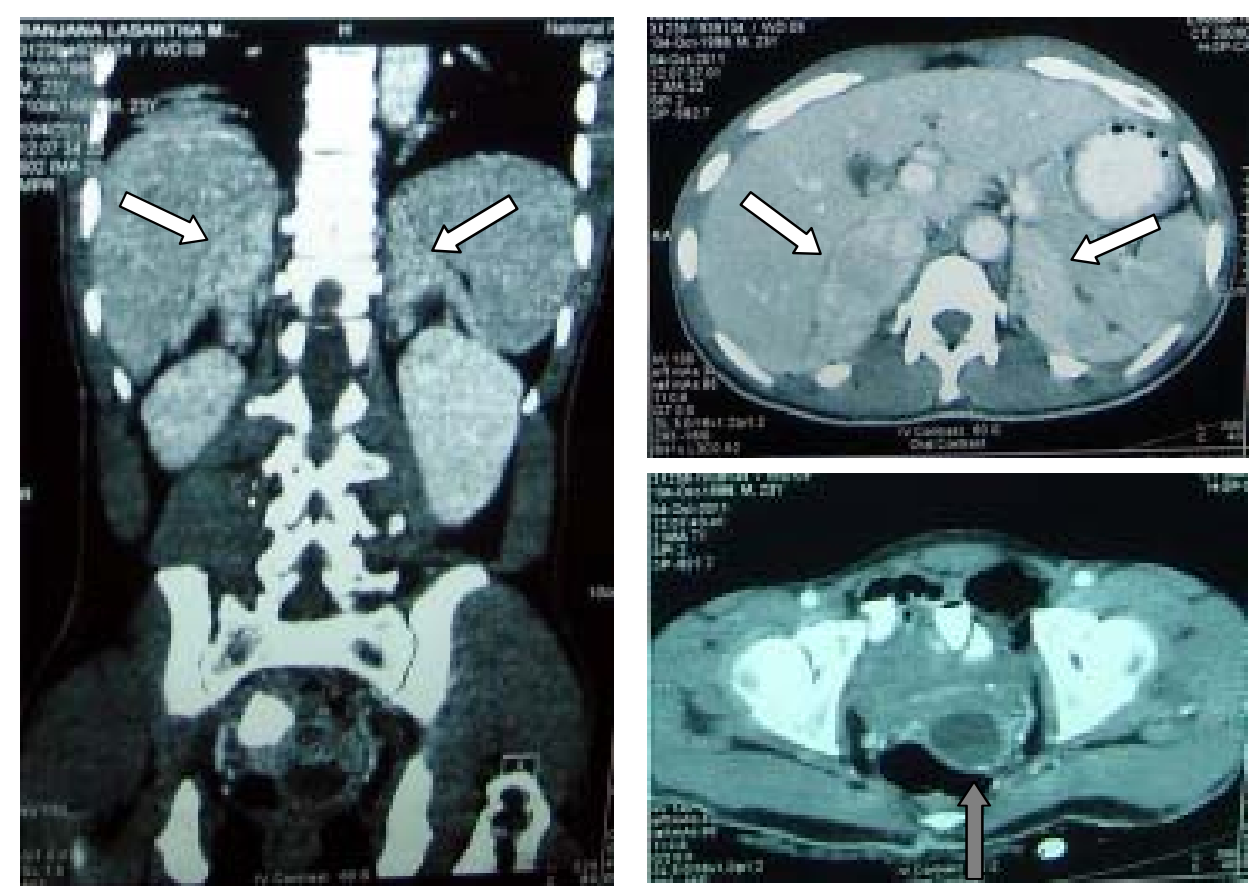

Figure $2 \mathrm{~A}$ and B - Bilateral adrenal hyperplasia (white arrows), C-Mullarian structures (grey arrow). 
karyotyping showed $46 \mathrm{XX}$ with negative SRY. A diagnosis of $11 \beta$ hydroxylase deficiency was made in view of hypertension with severe virilization in a $46 \mathrm{XX}$ individual. However the serum cortisol level in this patient was elevated (1232 and $868 \mathrm{nmol} / \mathrm{L}$ ) with unsuppressed overnight dexamethasone suppression test (ODST 102 $\mathrm{nmol} / \mathrm{L}$ ). Dehydroepiandrosterone sulfate (DHEAS) level was also within the normal range $(136 \mu \mathrm{g} / \mathrm{dL}-95 \%$ reference range: $35-430 \mu \mathrm{g} / \mathrm{dl})$. His urinary vanillylmandelic acid (VMA) level was $9.6 \mathrm{mg} / 24 \mathrm{hrs}$. (Ref: $1-11 \mathrm{mg} / 24 \mathrm{hrs}$.) After psychological counseling and education of the patient and the family, he chose to remain as a male. He was treated with nifedipine slow releasing tablet $20 \mathrm{mg}$ twice daily, low sodium diet to control hypertension, physiotherapy and speech therapy to improve motor skills and speech. Steroids, which are the mainstay of treatment in $\mathrm{CAH}$, were not started initially due to the negative effect they would have on androgen production and hence his male characteristics. However, he was advised to use steroids in stress situations and close monitoring with testosterone levels and 17-hydoxyprogesterone levels were planned on follow up visits. It was decided to start a long acting steroid if testosterone level remains markedly elevated during follow up. Urological referral was done in view of genital reconstruction and removal of mullerian structures once there is reasonable recovery from stroke.

\section{Discussion}

In $\mathrm{CAH}$ due to $11 \beta$ hydroxylase deficiency, development of the female external genitalia is affected in utero by excess fetal adrenal androgens, resulting in genitalia which are ambiguous. Most females with the severe or classical form of $\mathrm{CAH}$ are diagnosed in the neonatal period after presenting with ambiguous genitalia at birth or in early childhood. Occasionally, females are so severely virilized at birth that their external genitalia are male looking, with a penile urethra and fused labioscrotal folds (4). This leads to errors in gender assignment as in our patient. Due to delay in diagnosis in this patient, he was raised as a boy and didn't attend any regular medical follow up as he was seen as a normal male externally. The time which the initial diagnosis was suspected was too late for his parents to accept a new gender and he was lost to follow up. It has been shown that early diagnosis and treatment of CAH in a severally virilized female allows better psychological adjustment apart from preserving fertility (5). If the diagnosis is late, it is a dilemma whether to change the gender because of possible female fertility or to remove the female organs and continue to rear the child as a male. The decision depends on the age at diagnosis and the cultural context. Typical signs of androgen excess include masculinization of female external genitalia and precocious pseudopuberty in both sexes. Patients undergo rapid somatic growth with premature epiphyseal closure resulting in short adult stature. Elevated metabolites with mineralocorticoid activity, such as deoxycorticosterone and its derivatives, cause hypertension in about two thirds of patients $(4,6)$.
Biochemical profile and imaging suggested that $\mathrm{CAH}$ with $11 \beta$-hydroxylase deficiency is the most likely diagnosis in this patient. Generally in $\mathrm{CAH}$ patients, low cortisol levels increase ACTH production, flooding the adrenal steroidogenic machinery with upstream precursors (4). However in this patient cortisol levels were persistently elevated with non-suppressed levels following dexamethasone. Since other features were compatible with the diagnosis, this prompted us to think of an interference with the cortisol assay. Measurement of cortisol by immunoassay is compromised by the potential for cross-reactivity of reagent antibodies with structurally-related steroid compounds present in patient's serum (7). The elevated levels of cortisol in this patient was probably due to the elevated 11-deoxy cortisol levels cross reacting with the radioimmunoassay technique used in the estimation of cortisol level. Usually DHEAS is elevated in CAH, but some times there is only mild elevation and it is not a consistent feature (8). In our patient DHEAS level was within normal limits.

In this patient, abdominal imaging revealed preserved mullerian structures. Unlike the external genitalia, gonads and internal structures (ovarian tubes, uterus and cervix) that are derivatives of the Mullerian ducts are preserved since the substance that normally causes involution of these structures in men (mullerian inhibiting factor) is not produced by the foetal ovary (9).

Management of $\mathrm{CAH}$ due to $11 \beta$ hydroxylase deficiency comprises glucocorticoid administration to provide cortisol replacement and to normalize $\mathrm{ACTH}$, which in turn removes the drive for over secretion of deoxycorticosterone and in most cases brings about remission of hypertension. Patients with $11 \beta$-hydroxylase deficiency cannot mount a sufficient stress response and should receive appropriate stress doses of glucocorticoids as for other patients with adrenal insufficiency (4). In our patient, he and the family decided that he would remain as a male. The main challenge in this situation was to keep the androgen level to maintain his male characteristics and to control the hypertension. His blood pressure was controlled with nifedipine SR $20 \mathrm{mg}$ twice daily dose. It was decided not to start steroids initially as it would unmask his female characteristics such as regression of facial hair, male pattern of body hair, change of voice and initiation of menstrual periods which would present as urethral bleeding. It was targeted to keep the testosterone levels in the high normal range with regular monitoring. Steroid treatment was planned if the blood pressure control proves to be difficult or if the testosterone levels keep markedly elevated. Potassium-sparing diuretics, such as spironolactone is effective in controlling hypertension due to minaralocorticoid excess in $11 \beta$ hydroxylase deficiency. Spironolactone has antiandrogenic properties and would have unmasked the above mentioned female characteristics in our patient. His blood pressure was also adequately controlled with nifedipine and lifestyle 
modifications. Due to those reasons it was not started in our patient.

The patient was referred to the psychiatric unit for further counseling and he was explained about lack of fertility. Genitourinary referral was done for the correction of hypospadias and for the removal of mullerian structures. He gained slow but steady recovery from his limb weakness with continued physiotherapy.

Apart from karyotype analysis, DNA analysis is used routinely in some countries where common mutations have been identified. $11 \beta$-hydroxylase deficiency can now be diagnosed prenatally by measuring tetrahydro-11deoxycortisol in amniotic fluid (10). If one child in a family is already affected by congenital adrenal hyperplasia, prenatal diagnosis during pregnancy should be offered (11). In addition to enabling genetic counselling, prenatal diagnosis offers the opportunity to consider prenatal treatment with dexamethasone in order to prevent virilization of the external genitalia of XX fetuses (12). These genetic investigations are not available in the country.

Due to lack of availability, we were unable to perform plasma levels of 11-deoxycortisol and renin which would have conclusively proven the diagnosis, but as described earlier clinical and available biochemical results supported the diagnosis of CAH due to $11 \beta$-hydroxylase deficiency. Although ACTH dependent Cushing syndrome is a cause of bilateral adrenal hyperplasia especially when cortisol levels are high, lack of clinical features of Cushing syndrome and the overall clinical and biochemical picture with ambiguous genitalia was more in favour of CAH. Consistent with this diagnosis, ACTH level was thought to be high in this patient as evident by the body pigmentation. Biochemical measurement of ACTH was not undertaken due to unavailability at the time. Magnetic resonance imaging (MRI) of brain, to exclude an ACTH secreting pituitary adenoma was also not performed as it was not readily available and $\mathrm{CAH}$ was the most likely diagnosis.

\section{Conclusion}

We report this case to show the importance of early diagnosis, explanation and treatment in conditions which cause ambiguous genitalia in newborns including $\mathrm{CAH}$. Late diagnosis leads to gender reassignment at a age which is not acceptable to many patients and families causing psychological distress, loss of follow up and patients eventually present with complications of the disease. Patients and families need adequate psychological and social support and side effects of treatment should be monitored carefully.

\section{References}

1. Zachmann M, Tassinari D, Prader A. Clinical and biochemical variability of congenital adrenal hyperplasia due to 11 beta-hydroxylase deficiency. $J$ Clin Endocrinol Metab 1983; 56(2): 222-9.

2. White PC, Curnow KM, Pascoe L. Disorders of steroid 11 beta hydroxylase isozymes. Endocr Rev 1994; 15(4): 421-38.

3. Li-Qiang Zhao, Su Han, Hao-Ming Tian. Progress in molecular-genetic studies on congenital adrenal hyperplasia due to $11 \beta$-hydroxylase deficiency. World J Pediatr 2008; 4(2): 85-90.

4. Nimkarn S, New MI. Steroid 11 $\beta$-hydroxylase deficiency congenital adrenal hyperplasia. Trends Endocrinol Metab 2008; 19(3): 96-9.

5. Berenbaum SA, Korman Bryk K, Duck SC, Resnick SM. Psychological adjustments in children and adults with congenital adjustment in children and adults with congenital adrenal hyperplasia. J Pediatr 2004; 144 (6): 741-6.

6. Joehrer K, Geley S, Strasser-Wozak EM et al. CYP11B1 mutations causing non-classic adrenal hyperplasia due to $11 \beta$-hydroxylase deficiency. Hum Mol Genet 1997; 6(11): 1829-34.

7. Cook DM, Allen JP, Kendall JW, Swanson R. Interference of 21-Deoxycortisol with Cortisol Assay Methods. J Clin Endocrinol Metab 1973; 36(3): 608-10.

8. Turner HE, Wass JA. Congenital Adrenal Hyperplasia. Oxford handbook of Endocrinology and Diabetes, 2nd ed, Oxford University Press 2009: 306-307.

9. Tosatti Júnior R, Souza HS, Tosatti A. Congenital Adrenal Hyperplasia due to 11-Beta-hydroxylase Deficiency. Arq Bras Cardiol 2005; 85(6): 421-4.

10. Rösler A, Leiberman E, Rosenmann A, Ben-Uzilio R, Weidenfeld J. Prenatal diagnosis of 11 beta-hydroxylase deficiency congenital adrenal hyperplasia. J Clin Endocrinol Metab 1979; 49(4): 546-51.

11. Deaton MA, Glorioso JE, McLean DB. Congenital Adrenal Hyperplasia: Not Really a Zebra. Am Fam Physician 19991; 59(5): 1190-6, 72.

12. Cerame BI, Newfield RS, Pascoe L, et al. Prenatal diagnosis and treatment of 11 beta-hydroxylase deficiency congenital adrenal hyperplasia resulting in normal female genitalia. J Clin Endocrinol Metab 1999; 84(9): 3129-34. 